\title{
ROTATIONAL CHARACTERISTICS OF CORONAL HOLES
}

\author{
WILLIAM J. WAGNER \\ Sacramento Peak Observatory, AFCRL, Sunspot, N.M. 88349, U.S.A.
}

\begin{abstract}
From May 1972 to October 1973, daily measures were obtained of EUV coronal hole areas appearing at the central meridian. Autocorrelations of these coronal hole area time series provide synodic rotation periods which indicate an almost rigid rotation by such features for lag lengths as short as one rotation. The rotation periods of coronal holes at high latitudes best compare with inferred interplanetary field rotation periods.
\end{abstract}

In view of the complexity of the rotational characteristics of solar phenomena and because of the possible link between coronal holes and large-scale magnetic field structure, it seems important to determine the rotational properties of coronal holes.

Daily spectroheliograms were obtained in $\lambda 284$ of Fe XV by R. D. Chapman, W. M. Neupert, and R. J. Thomas of the Goddard Space Flight Center using OSO 7. These appear as intensity isophote maps in Solar-Geophysical Data, issued by the Environmental Data Service of NOAA, and serve as the basic data used in this work. I have arbitrarily chosen an EUV count level of less than 10 as a brightness level which defines a coronal hole. This threshold was allowed to drop to 5 or rise to 20 depending on the ratio of total EUV counts to $10-\mathrm{cm}$ radio flux for each particular day (Chapman and Neupert, 1974). No figure of merit was applied for the 'depth' of the coronal hole: rather, a binary division of the spectroheliogram was made into either hole or non-hole areas.

Seven latitude zones were studied: $80^{\circ}-60^{\circ} \mathrm{N}, 60^{\circ}-40^{\circ} \mathrm{N}, 40^{\circ}-20^{\circ} \mathrm{N}, 20^{\circ} \mathrm{N}-20^{\circ} \mathrm{S}$, $20^{\circ}-40^{\circ} \mathrm{S}, 40^{\circ}-60^{\circ} \mathrm{S}, 60^{\circ}-80^{\circ} \mathrm{S}$. Figure 1 a shows one typical $\lambda 284$ isophote map

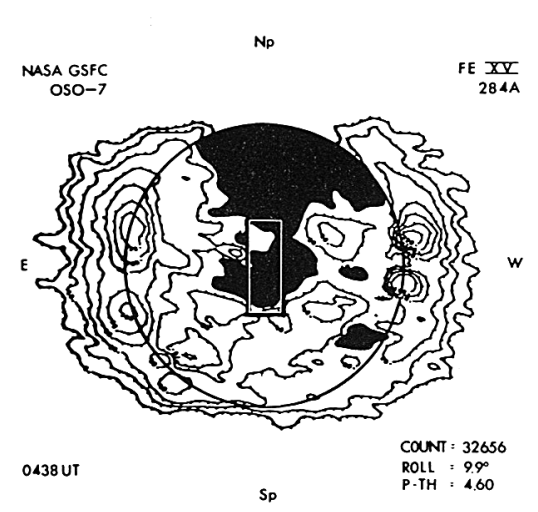

(a)

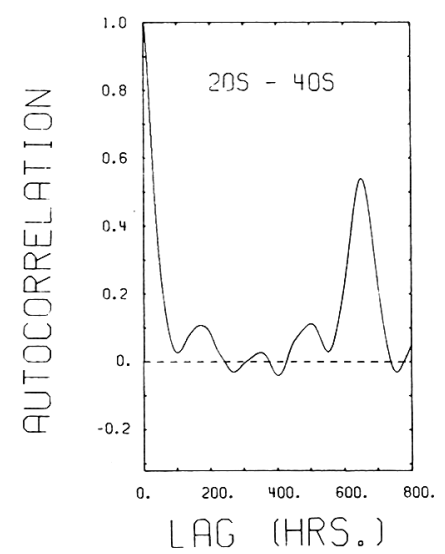

(b)

Figs. 1a-b. The data from which rotation periods were derived appears in Solar-Geophysical Data of NOAA. (a) On daily OSO-7 isophote maps, coronal hole regions were identified (dark shading). Apertures of $14^{\circ}$ width in each latitude zone were used in measuring coronal hole area at the central meridian. (b) The autocorrelation function for the $20^{\circ}-40^{\circ} \mathrm{S}$ coronal hole area series. The location of the peak near $650 \mathrm{~h}$ marks the synodic rotation period. Isophote map from World Data Center A, NOAA. 
with an imaginary aperture one rotation day $\left(14^{\circ}\right)$ in width straddling the central meridian. The coronal hole area in each of the seven latitude zones was recorded with these apertures each day from 1 May 1972, through 31 October 1973.

Using such time series of coronal hole area, autocorrelation functions were generated for each of the seven latitude zones, as shown in Figure 1b. The major peak near a lag of $650 \mathrm{~h}$ marks the recurrence of those coronal hole features which persisted for at least one synodic rotation period. The zonal synodic rotation periods thus derived are shown in Figure 2. The error bars represent standard deviation from the mean of similar rotation periods obtained by subdividing the 18 -month time series into four data subsets of 4.5 months each.

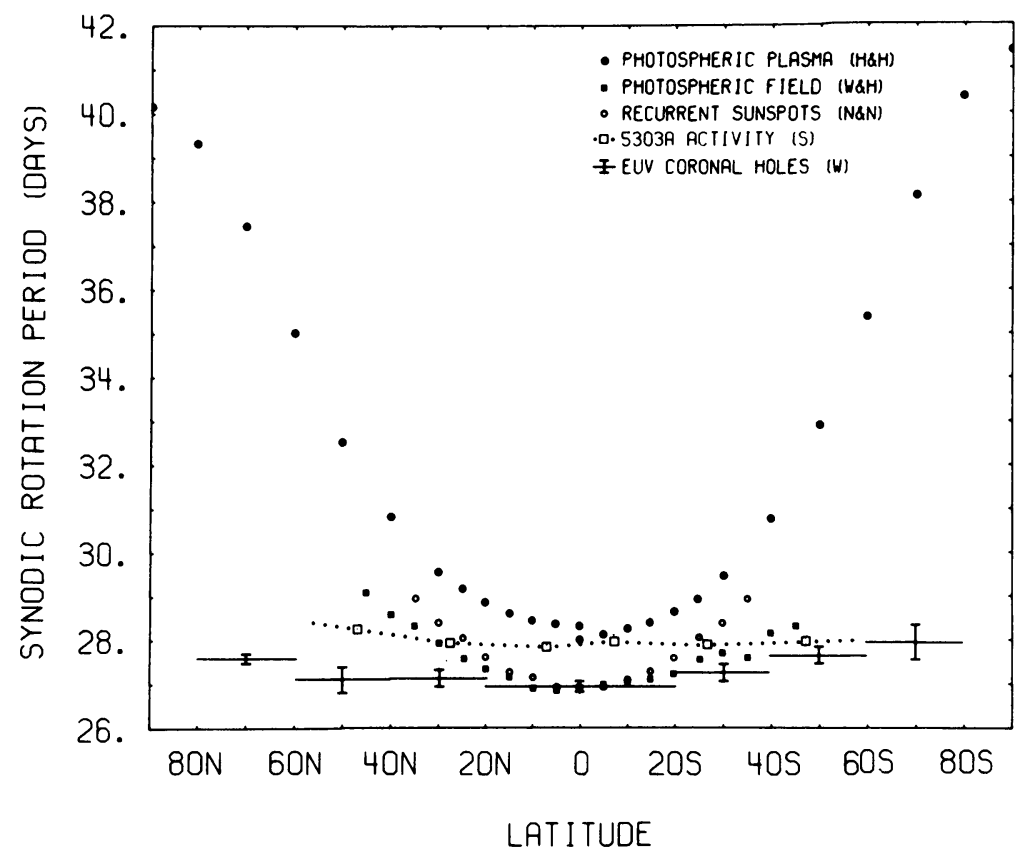

Fig. 2. EUV coronal hole rotation rates from the present work. Low-emissivity coronal holes and active green line coronal structures (Sykora, 1971) perform almost rigid rotation when contrasted to: photospheric plasma (Howard and Harvey, 1970); photospheric magnetic fields (Wilcox and Howard, 1970); and the classical differential rotation (Newton and Nunn, 1951) of recurrent sunspots (with alterations from Wagner, 1975).

In Figure 2, it may be seen that coronal holes do not participate in the differential rotation exhibited by sunspots (Newton and Nunn, 1951), photospheric magnetic fields (Wilcox and Howard, 1970), or photospheric plasma (Howard and Harvey, 1970). While equatorial rotation periods are generally comparable, at higher latitudes considerable slippage of fields and plasma occurs with respect to the coronal holes. This near-rigid rotation of these low-emissivity coronal hole features is quite similar to that reported earlier by Sykora (1971) for bright coronal green line structure up to latitudes $\pm 57^{\circ} .5$. I find that, for the corona in 1972-1973, the 
northern hemisphere rotated marginally faster than the southern, contrary to the 1947-1968 results of Sykora (1971).

Now, if bright coronal features correspond to centers of activity, then coronal holes may be said to represent inactivity. Analogously, during these 18 months, a rather consistent pattern was observed in these data of 'inactive longitudes'. This pattern was especially evident in the low $\left(\leq 40^{\circ}\right)$ latitude regions, and consisted of four orthogonal longitudes which were consistently favored by coronal holes.

Altschuler et al. (1972) have provided a description of coronal holes as regions of diverging magnetic field open to interplanetary space. From May 1972 to October 1973 , the mean rotation period of the interplanetary field patterns (with field directions inferred by the method of Svalgaard, 1972) was somewhat greater than 28 days. If coronal holes are to be related to interplanetary fields at $1 \mathrm{AU}$, the results of the present work imply that the polar region coronal holes with 28 -day rotation periods are more pertinent features than low latitude holes with 27-day periods.

In conclusion, coronal holes show almost rigid rotation. At low latitudes during this period, four orthogonal 'inactive longitudes' existed which showed coronal holes. Coronal hole rotation periods at high latitudes best compare with inferred interplanetary field rotation periods.

\section{References}

Altschuler, M. D., Trotter, D. E., and Orrall, F. Q.: 1972, Solar Phys. 26, 354.

Chapman, R. D. and Neupert, W. M.: 1974, J. Geophys. Res. 79, 4138.

Howard, R. and Harvey, J.: 1970, Solar Phys. 12, 23.

Newton, H. W. and Nunn, M. L.: 1951, Monthly Notices Roy. Astron. Soc. 111, 413.

Svalgaard, L.: 1972, J. Geophys. Res. 77, 4027.

Sykora, J.: 1971, Solar Phys. 18, 72.

Wagner, W. J.: 1975, Astrophys. J. Letters 198, L141.

Wilcox, J. M. and Howard, R.: 1970, Solar Phys. 13, 251.

\section{DISCUSSION}

Stix: Which latitude of the coronal hole exactly corresponds to the rotation of the interplanetary sector structure?

Wagner: I would have to say latitudes higher than about $\pm 50 \mathrm{deg}$. Certainly not the equatorial or low latitudes.

Stenfio: The result that coronal holes appear to rotate rigidly agrees with the observation that the background magnetic fields have a rotation more similar to rigid rotation than fields in active regions. As magnetic fields reflect rotation rates in deeper layers, and as they rotate faster than the photospheric plasma, the angular velocity of rotation should increase with depth. 